\title{
How is the Toxicity of Heavy Metals Detected in the Problems of Metal-Metal Hip Arthroplasties?
}

\author{
Fernandez-Ibanez A*1, Ugalde-Herra R${ }^{2}$, Rodriguez-Getino JA ${ }^{1}$, Garcia-Casas JB ${ }^{1}$, Diaz-Suarez JC ${ }^{2}$ \\ ${ }^{1}$ Department of Medicine, University of Oviedo, Spain
}

${ }^{2}$ Hospital Universitario Central de Asturias, Oviedo (Asturias), Spain

Received: June 13, 2018; Published: June 21, 2018

*Corresponding author: Fernandez-Ibanez A, Department of Medicine, University of Oviedo, Spain

\begin{abstract}
Introduction: Total hip arthroplasties are the most frequent interventions in elderly patients. In some cases, the presence of metal residues in routine analytical serum may be an indicator of the onset of prosthetic wear, although this presence does not mean that it needs urgent replacement.
\end{abstract}

Objective: To detect the patients with joint inflammatory symptoms in their serum have ranges of toxicity in heavy metals.

Method: Review of the clinical history of patients who meet the characteristics of joint inflammation and determination of heavy metals. Collection of blood samples for processing by mass spectrometry with induced coupling plasma, which is the reference method.

Results: Differentiation in 3 groups of patients according to the trace element analyzed and its own toxicological reference limit.

Conclusion: The concentrations of heavy metals cannot be analyzed in isolation, they will be interpreted in the overall clinical-pathological context of the patient under study.

Keywords: Heavy Metals; Hip Arthroplasties; Toxicity

\section{Introduction}

Patients subjected to total metal-metal hip arthroplasties, in some cases, present different intraoperative complications such as fractures, peripheral nerve injury, vascular damage or hypotension and other long-term complications after surgery, such as thromboembolic disease, infection, dislocations, osteolysis and wear of the components, aseptic mobility, periprosthetic fractures, dismetrias or definitive failure by mobilization of the prosthetic components [1-4]. The appearance of heavy metals in serum can be an indicator of the beginning of the wear of a prosthesis, although not always the appearance of an elevation of prosthetic metals in serum means that the implant does not work correctly. The presence of metal debris can be due to several factors, since in the total hip arthroplasties the existence of several components involves friction surfaces that generate metallic, ceramic or polyethylene wastes; Most waste comes from the friction surface.

They are also generated at the interface of the femoral stem with bone or with cement, particularly when the prosthesis is loosened, and the metals may come from the acetabular metal component and its fixing surface with screws, cerclage wires, plates and screws or dental implants or heart valves. Metals can be produced by delamination of the metal surface and it cannot be forgotten that when the patient's renal function is altered the elimination by metals of urine decreases [5-8]. Some prosthetic revision surgeries have seen deposits of metallic particles, near the prosthesis or in distant tissues, which imply an electrochemical interaction with the surrounding tissue fluids and, although they have decreased in recent years, metallic corrosions can be found. in the replaced components, as well as degradation products that stimulate osteolysis by the activation of macrophages and the acceleration of polyethylene wear. To find solutions to this problem, especially in young patients, in recent years new polyethylenes have been introduced in the designs and the metal-metal friction pair has resurfaced [9-15].

It is known that some models of these arthroplasties can produce clinical alterations derived from the presence of heavy metals in the organism (blood and urine) although currently there are no known carcinogenic or fetal complications, if local alterations can occur in the form of cysts or pseudocysts called ALVAL (aseptic lymphocyte-dominant vasculitis-associated lesion) and systemic 
as hepatic or renal alterations. In this study, the concentrations of heavy metals (chromium, cobalt and titanium) in serum of patients subjected to total metal-metal hip arthroplasty with inflammatory symptoms or clinical signs susceptible to problems at the joint level were measured to determine the concentrations of the products released in these processes. Different follow-up protocols have been published with guidelines on the normal figures of ions in blood and urine, on the maximum admissible levels and on the convenience of a necessary monitoring [16-18]. These analytical determinations require special care in the collection and transfer of samples and are not routinely performed in the laboratories of general hospitals. They can also be altered occasionally by the intake of food products, by the contribution of medications or environmental situations.

\section{Patients and Methodology}

The criteria for the inclusion of patients were of age undergoing primary total hip arthroplasty, with etiology of coxarthrosis, who did not have a contralateral prosthesis or dental implants and who presented inflammatory signs or symptoms compatible with prosthetic problems during follow-up. In the selected period for the development of work (2015), 19 postoperative follow-ups were performed. Samples were collected from 6 women and 13 men, aged between 36 and 78 years. The venous blood samples were obtained in vacuum tubes free of trace elements and without anticoagulants. They were centrifuged at 10,000 rpm, for 10 minutes, separated and frozen at $-80^{\circ} \mathrm{C}$ until processing.

The trace elements were all analyzed according to the reference method that is mass spectrometry with induced coupling plasma (7700 series ICP-MS Agilent Technologies $®$ ) that consists in generating a plasma (gas strongly ionized, electrically neutral but current conductor) by subjecting a flow of argon gas to the action of an oscillating magnetic field induced by a high frequency current. In the interior of the plasma temperatures of $6000-10000^{\circ} \mathrm{C}$ can be reached; Under these conditions, the atoms present in the sample are ionized. The sample in liquid form is transported by means of a peristaltic pump to the nebulizer system, where it is transformed into an aerosol thanks to the action of argon gas. This aerosol is transported to the plasma where it is vaporized, atomized and excited / ionized.

The ions generated in the plasma pass through a quadrupole filter, which separates the ions generated in the plasma according to their mass/charge ratio $(\mathrm{m} / \mathrm{z})$. Each of the separated masses reaches the detector where the sample element is quantified. Extra precautions should be taken in the extraction to avoid the presence of contamination in the place of collection of the sample and of the specimens themselves. The use of procedures or devices that are not free of trace elements will cause falsely increased results since titanium and cobalt are very common metals in the environment. In the case of chromium, anticoagulants should be avoided in the extraction tubes because they have traces of this metal.

\section{Results}

For the determination of these trace elements it is necessary to avoid the consumption of mineral supplements in the 5 days prior to taking the sample and not quantify them if radiological contrasts of gadolinium, iodine or barium are administered until 4 days have elapsed from said administration. The interpretation of the results depends on the element analyzed and its reference limit indicating toxicity, for which the patients have been divided into 3 groups:

\section{First group}

Titanium (n=6). An increase in the serum concentration of the metal above $10 \mu \mathrm{g} / \mathrm{L}$ is indicative of deterioration of the prosthesis. It must be taken into account, in the absence of clinical information to corroborate the finding, a high serum concentration of titanium is not an independent predictor variable of failure or deterioration of the prosthesis. Only one of the patients resulted in $18 \mu \mathrm{g} / \mathrm{L}$ and had markers of inflammation and acute infection, which confirmed the clinical suspicion of prosthetic deterioration. The rest of the patients had levels between $1.70 \mu \mathrm{g} / \mathrm{L}$ and $4.9 \mu \mathrm{g} / \mathrm{L}$.

\section{Second Group}

Cobalt $(n=5)$. In patients with total hip prosthesis with a metalmetal friction torque that have levels higher than $10 \mu \mathrm{g} / \mathrm{L}$, they are indicative of wear of the prosthesis. The quantification of cobalt should not be used to evaluate the activity of vitamin B12. Patients with concentrations of $14.3 \mu \mathrm{g} / \mathrm{L}$ and $16 \mu \mathrm{g} / \mathrm{L}$ showed signs of infection in their analytes due to high acute phase reactant markers, so antibiotics and follow-up were scheduled in 2-3 months. The patient with concentrations of $27 \mu \mathrm{g} / \mathrm{L}$ was operated on urgently for a prosthetic replacement and followed later with routine analytical controls to observe its correct evolution. The remaining patients had analytical values between $5.8 \mu \mathrm{g} / \mathrm{L}$ and $9 \mu \mathrm{g} / \mathrm{L}$.

\section{Third Group}

Chrome $(n=15)$. In patients with total hip prosthesis with a metal-metal friction torque that have levels above the reference limit of $1 \mu \mathrm{g} / \mathrm{L}$, they are indicative of prosthetic wear. In the latter case, there are several patients $(n=8)$ with inflammatory processes and prostheses in poor condition that need replacement since the chromium concentrations are between $1.2 \mu \mathrm{g} / \mathrm{L}$ and $4.4 \mu \mathrm{g} / \mathrm{L}$ and there are clinical-analytical evidences that support this assumption.

\section{Discussion and Conclusions}

It should be considered that $\mathrm{Cr}$ and Co are essential elements for human life and are present in healthy people, being able to consider the 95 th percentile of the distribution $(0.3 \mu \mathrm{g} / \mathrm{L}$ for chromium and $1 \mu \mathrm{g} / \mathrm{L}$ for cobalt) as a possible reference value in healthy people. In several of the patients analyzed, clinical suspicion of poor evolution or wear of the prosthesis that required urgent replacement was described. Cobalt is a cofactor of vitamin B12 and chromium is an essential cofactor in the interaction between insulin and its receptor, but both are toxic at sufficiently high concentrations in the body. Hexavalent chromium is a possible product of degradation of the implant; in 1990 it was classified as a class I carcinogen for humans by the American Agency for Research on Cancer. Titanium and cobalt have shown carcinogenic activity in animals, but they have not been investigated in humans. High concentrations of cobalt can induce polycythemia, testicular toxicity and interfere 
with DNA repair mechanisms, but no causal relationship has been definitively established in people with total hip replacement.

The most used prostheses have been those of titanium and some clinical presentations compatible with metallic toxicity have been observed. Although in Spain there are studies of the metallic composition of the periprosthetic interface, there are few data published in our country on the expected serum concentrations in blood of patients undergoing total hip arthroplasty. In particular, there is little data on titanium, a very refractory element, and its determination in the clinical environment is usually not available. The description of the values can be very useful for the definition of epidemiological databases, to continue developing the proposed line of research and to give a quick response to problems such as defective prostheses placed lately. The quantification of these elements is difficult because the reference method is not always available for detection in the clinical environment, both because of its high cost and because of the need for qualified personnel, which sometimes implies a delay in the diagnosis of the patient. Most studies agree that heavy metal concentrations should not be used in isolation to assess the patient if they are not interpreted in the appropriate clinical context.

\section{References}

1. Thien TM, Karrholm J (2010) Design-related risk factors for revision of primary cemented stems. Acta Orthop 81(4): 407-412.

2. Rasquinha V, Chitranjan R, Weiskopf J, Rodríguez J, Skipor A, et al. (2006) Serum metal levels and bearing surfaces in total hip arthroplasty. Arthroplasty 2(6): 47-52.

3. Wayne, Pennsylvania NCCLS (1997) Control of Preanalytical Variation in Trace Element Determinations Approved Guideline. NCCLS document C38-A (ISBN 1-56238-332-9). NCCLS, 940, Suite 1400,19087, West Valley Road, Pakistan.

4. Wagner P, Olsson H, Ranstam J, Robertsson O, Hao Zheng M, et al. (2012) Metal-on-metal joint bearings and hematopoetic malignancy. Acta Orthopaedica 83(6): 553-538.

5. Willert HG, Buchhorn GH, Fayyazi A, Flury R, Windler M, et al (2005) Metal-on-metal bearings and hypersensitivity in patients with artificial hip joints. clinical and histomorphological study 87(1): 28-36.

6. Kwon YM, Ostlere SJ, McLardy Smith P, Athanasou N, Gill HS, et al. (2011) Asymptomatic pseudotumors after metal-on-metal hip resurfacing arthroplasty: Prevalence and metal ion study. 26(4): 511-518.
7. Kwon YM, Lombardi AV, Jacobs JJ, Fehring TK, Lewis CG, Cabanela ME (2014) Risk stratification algorithm for management of patients with metal-on-metal hip arthroplasty. Consensus statement of the American Association of Hip and Knee Surgeons, the American Academy of Orthopaedic Surgeons, and The Hip Society 96(1): e4 (1-6).

8. Jacobs JJ, Skipor AK, Patterson LM, Hallab NJ, Paprosky P (1998) Metal release in patients who have a primary total hip arthroplasty. A prospective, controlled, longitudinal study. J Bone Joint Surg (Am) 80(10): 1447-1458.

9. Dorr LD, Bloebaum R, Emanuel J, Meldrum R (1990) Histological biochemical and ion analysis of tissue and fluids retrieved during total hip arthroplasty. Clin Orthop Rel Res 261: 82-95.

10. Jacobs J, Skipor A, Black J (1991) Metal release and excretion in patients with titanium-base alloy total hip replacement components. J Bone Joint Surg (Am) 73(10): 1475-1479.

11. Leopold S, Berger R, Patterson L, Skipor A, Urban R (2000) Serum titanium level for diagnosis of a failed, metal-backed patellar component. J Arthroplasty 15(7): 938-943.

12. Jacobs J, Skipor A, Campbell P, Hallab N, Urban R, et al. (2004) Can metal levels be used to monitor metal-on metal hip arthroplasties. J Arthroplasty 19(8): 59-65.

13. Goullé JP, Mahieu L, Castermant J, Neveu N, Bonneau L, et al. (2005) Metal and metalloid multi-elementary ICP-MS validation in whole blood, plasma, urine and hair. Reference values. Forensic Sci Int. 153(1): 39-44.

14. Iyengar V, Woittiez J (1988) Trace elements in human clinical specimens: Evaluation of literature data to identify reference values. Clin Chem 34(3): 474-481.

15. Sampson B, Hart A (2012) Clinical usefulness of blood metal measurements to assess the failure of metal-on-metal hip implants. Ann Clin Biochem 49(2): 118-131.

16. Jonathon R Campbell, Mathew P Estey (2013) Metal release from hip prostheses: cobalt and chromium toxicity and the role of the clinical laboratory. Clin Chem Lab Med 51(1): 213-220.

17. Smolders JM, Bisseling P, Hol A, Van Der Straeten C, Schreurs BW, et al. (2011) Metal ion interpretation in resurfacing versus conventional hip arthroplasty and in whole blood versus serum. How should we interpret metal ion data? Hip Int 21(5): 587-595.

18. Newton AW, Ranganath L, Armstrong C, Peter V, Roberts NB (2012) Differential distribution of cobalt, chromium, and nickel between whole blood, plasma and urine in patients after metal-on-metal (mom) hip arthroplasty. J Orthop Res 30(10): 1640-1646.
This work is licensed under Creative Commons Attribution 4.0 License

Submission Link: https://biomedres.us/submit-manuscript.php

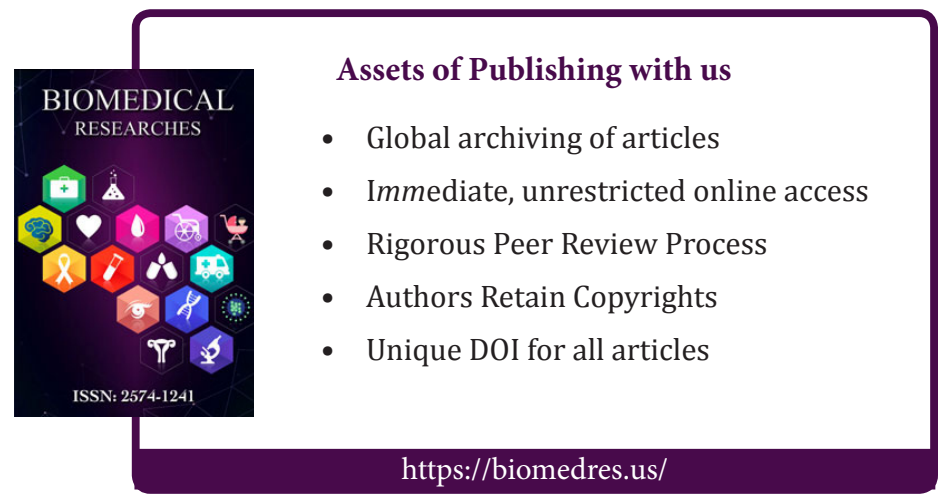

